\title{
Low-melting zirconium alloys
}

\author{
A. M. Savchenko ${ }^{\dagger}$, Y. V. Konovalov, A. V. Laushkin, O. I. Yuferov \\ ${ }^{\dagger}$ sav-alex111@mail.ru
}

Stock Company «A.A. Bochvar High-Technology Research Institute of Inorganic Materials» (SC VNIINM) VNIINM, 123060, Moscow, Russia

\begin{abstract}
A novel class of low-melting Zr-based alloys has been developed. These are deep triple and quadruple eutectics with melting points from 690 to $860^{\circ} \mathrm{C}$, which are very low for zirconium. Projections of the crystallization areas in the $\mathrm{Zr}$-rich corner of $\mathrm{Zr}-\mathrm{Be}-\mathrm{Fe}$ and $\mathrm{Zr}-\mathrm{Cu}-\mathrm{Fe}$ systems are given. Apart from eutectic zirconium alloys, hypoeutectic alloys, in which the melting point changes not significantly, but the ductility is greatly enhanced, can also be used. An important feature of these alloys is the possibility of additional alloying up to 3 to 5 mass per cent retaining the low melting temperature. As alloying elements, $\mathrm{Nb}, \mathrm{Cu}, \mathrm{Al}, \mathrm{Mo}$, Si etc. can be used. The additional alloying can be done to enhance the corrosion resistance, castability, capillary properties and improve the neutron-physics characteristics. The temperature coefficient of linear expansion and thermal conductivity of alloys is higher than that of zirconium. Corrosion resistance in water and superheated steam remained high, characteristic of zirconium alloys. Capillary properties of alloys are very high, so they can be used as cast ones as well as a solder for brazing of different materials. These alloys can be also produced in an amorphous state as granules and strips. They can be used as hydrides for hydrogen accumulation too. It is promising to use the low-melting Zr-based alloys proposed in nuclear engineering as matrix materials for dispersion fuel elements. The fuel compositions including these alloys have a high thermal conductivity and compatibility and uranium level 30 to $50 \%$ higher than in the fuel elements of VVER and PWR reactors. The use of new dispersion fuel can lead to improved neutron-physical characteristics of the reactors, increase burnout, reduce fuel temperature and improve fuel efficiency.
\end{abstract}

Keywords: Zr-base alloys, low-melting alloys, eutectics, atomic energy, fuel element.

\section{Легкоплавкие циркониевые сплавы}

\author{
Савченко А. М. ${ }^{\dagger}$, Коновалов Ю. В., Лаушкин А. В., Юферов О.И. \\ АО «Высокотехнологический научно-исследовательский институт неорганических материалов \\ имени академика А.А. Бочвара» (АО «ВНИИНМ»), 123060, г. Москва, Россия
}

Разработан новый класс легкоплавких циркониевых сплавов. Они представляют собой глубокие тройные и четверные эвтектики с очень низкими для циркония температурами плавления от 690 до $860^{\circ} \mathrm{C}$. Приведены проекции поверхностей кристаллизации циркониевого угла систем цирконий-бериллий-железо и цирконий-медь-железо. Помимо эвтектических циркониевых сплавов можно также использовать сплавы доэвтектических составов, в которых температура плавления меняется незначительно, зато резко возрастает пластичность сплавов. Важной особенностью этих сплавов является возможность их дополнительного легирования до 3 - 5\% масс. при сохранении низкой температуры плавления сплавов. В качестве легирующих элементов могут использоваться $\mathrm{Nb}, \mathrm{Cu}, \mathrm{Al}, \mathrm{Mo}, \mathrm{Si}$ и т. д. Дополнительное легирование может производиться для повышения коррозионной стойкости, жидкотекучести, капиллярных свойств, улучшения нейтронно-физических характеристик. Температурный коэффициент линейного расширения и теплопроводность сплавов выше, чем у нелегированного циркония. Коррозионная стойкость в воде и перегретом паре высоких параметров осталась высокой, свойственной циркониевым сплавам. Сплавы обладают высокими капиллярными свойствами, могут использоваться как литейные, могут применяться для пайки разнородных материалов, могут использоваться в аморфном состоянии в виде гранул и лент. Возможно также их использование как гидридов для аккумуляции водорода. Перспективно использование легкоплавких циркониевых сплавов в атомной энергетике в качестве матричных сплавов дисперсионных твэлов. Топливные композиции, включающие эти сплавы, обладают с высокой теплопроводностью, совместимостью и ураноемкостью на 30 - $50 \%$ выше, чем для твэлов реакторов ВBЭР и PWR. Использование нового дисперсионного топлива может привести к улучшению нейтронно-физических характеристик реакторов, увеличить выгорание, снизить температуру топлива и повысить работоспособность топлива в режиме переменных нагрузок.

Ключевые слова: циркониевые сплавы, легкоплавкие сплавы, эвтектика, атомная энергетика, тепловыделяющий элемент. 


\section{1. Введение}

Циркониевые сплавы широко применяются в атомной энергетике в качестве конструкционного материала в активных зонах реакторов на тепловых нейтронах, в первую очередь, в качестве оболочек твэлов [1-3].

Использованию циркониевых сплавов в качестве матричных сплавов дисперсионных тепловыделяющих элементов препятствует относительно высокая температура плавления этих сплавов (температура плавления циркония $1852^{\circ} \mathrm{C}$ ), тогда как методы пропитки, используемые для изготовления дисперсионных тепловыделяющих применимы только для матричных сплавов с температурой плавления не выше $900^{\circ} \mathrm{C}$. Поэтому требовалось разработать новые сплавы с относительно низкими температурами плавления (до $900^{\circ} \mathrm{C}$ ).

Ранее были разработаны несколько классов новых циркониевых сплавов $[4,5]$. В настоящей работе проводится анализ возможностей и особенностей использования этих сплавов и их модификаций.

\section{2. Состав и структура легкоплавких циркониевых сплавов}

Указанные циркониевые сплавы $[4,5]$ представляют собой глубокие тройные и четверные эвтектики (см. рис. 1) с очень низкими для циркония температурами плавления от 690 до $860^{\circ} \mathrm{C}$. Базовые составы сплавов приведены в табл. 1.

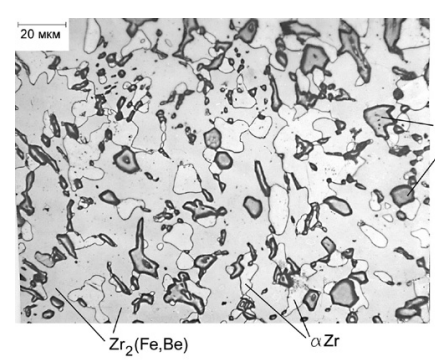

a

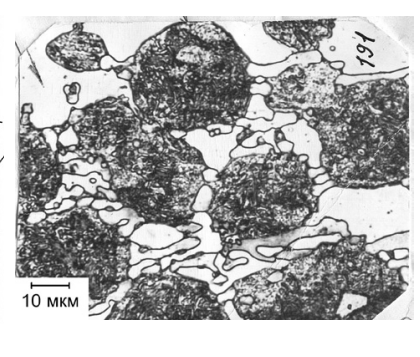

b
Рис. 1. Микроструктура сплавов систем: Zr-Fe-Be (a); доэвтектический $\mathrm{Zr}-\mathrm{Fe}-\mathrm{Cu}(\mathrm{b})$.

Fig. 1. Microstructure of alloys of systems: Zr-Fe-Be (a); hypoeutectic $\mathrm{Zr}-\mathrm{Fe}-\mathrm{Cu}$ (b)

Табл. 1. Составы и температуры плавления легкоплавких циркониевых сплавов.

Table 1. Compositions and melting temperatures of low-melting zirconium alloys.

\begin{tabular}{|c|c|c|c|c|c|}
\hline $\begin{array}{c}\text { Номер } \\
\text { группы } \\
\begin{array}{c}\text { Group's } \\
\text { number }\end{array}\end{array}$ & \multicolumn{4}{|c|}{$\begin{array}{c}\text { Содержание легирующих } \\
\text { элементов, \% масс. } \\
\text { Composition of alloy, \% mass }\end{array}$} & $\begin{array}{c}\text { Температура } \\
\text { плавления, } \\
\text { Melting } \\
\text { temperature, }\end{array}$ \\
\hline & $\mathrm{Ti}$ & $\mathrm{Fe}$ & $\mathrm{Cu}$ & $\mathrm{Be}$ & \\
\hline 1 & - & $6-12$ & $6-12$ & - & $850-860$ \\
\hline 2 & - & $4-8$ & - & $2-3$ & $780-810$ \\
\hline 3 & $5-10$ & $8-14$ & $8-14$ & - & $810-820$ \\
\hline 4 & $5-20$ & $4-7$ & $1-3$ & $1.5-2.5$ & $690-720$ \\
\hline
\end{tabular}

Важной особенностью этих сплавов является возможность их дополнительного легирования до 3-5\% масс. при сохранении низкой температуры плавления сплавов. В качестве легирующих элементов могут использоваться $\mathrm{Nb}$ (для повышения коррозионной стойкости), $\mathrm{Cu}$ и $\mathrm{Al}$ (для улучшения жидкотекучести и капиллярных свойств, для понижения температуры плавления), Мо и $\mathrm{Si}$ (для улучшения механических характеристик) и т.д.

Сплавы групп 1 и 2 предназначены для использования в тепловых атомных реакторах, групп 3 и $4-$ в быстрых реакторах. Добавка титана к сплавам групп 3 и 4 улучшает совместимость и сцепление матричных сплавов со стальными оболочками, которые применяются в тепловыделяющих элементах быстрых реакторов.

В структуре сплавов основной фазой является фаза типа $\mathrm{Zr}_{2}(\mathrm{Fe}, \mathrm{X})$ с объемно-центрированной тетрагональной кристаллической решеткой, где $\mathrm{X}$ - это элементы $\mathrm{Cu}, \mathrm{Be}, \mathrm{Ni}, \mathrm{Nb}, \mathrm{Al}, \mathrm{Si}$ и т. д. Большая растворимость в данной фазе легирующих элементов приводит к возможности сохранения на высоком уровне положительных свойств сплавов, в частности, коррозионной стойкости, теплопроводности, литейных свойств, при варьировании легирующих элементов в широком диапазоне. Остальными фазами, в зависимости от составов сплавов являются $\alpha-\mathrm{Zr}, \mathrm{Zr}_{2} \mathrm{Cu}, \mathrm{ZrBe}_{2}$ и т.д. На рис. 2 приведены проекции поверхностей кристаллизации систем $\mathrm{Zr}-\mathrm{Fe}-\mathrm{Be}$ и $\mathrm{Zr}-\mathrm{Fe}-\mathrm{Cu}$, в табл. 2 указаны нонвариантные реакции в этих системах. Элементы $\mathrm{Fe}, \mathrm{Be}, \mathrm{Cu}$ вместе с цирконием составляют основу первой и второй групп сплавов.

Следует отметить, что помимо эвтектических циркониевых сплавов можно также использовать сплавы доэвтектических составов (см. рис. 1b), в которых температура плавления меняется незначительно, зато резко возрастает пластичность сплавов.

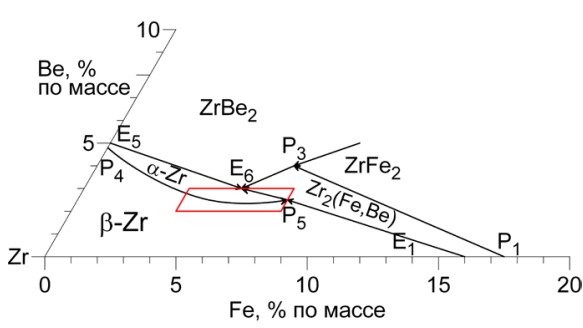

a

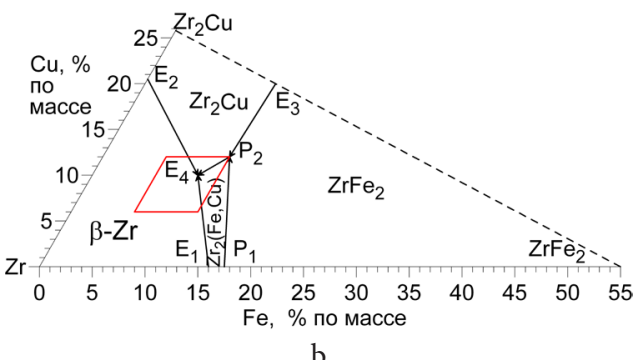

Puc. 2. (Color online) Проекции поверхностей кристаллизации циркониевого угла систем $\mathrm{Zr}-\mathrm{Be}-\mathrm{Fe}$ (a) и $\mathrm{Zr}-\mathrm{Cu}-\mathrm{Fe}$ (b). Красным цветом выделена область составов легкоплавких сплавов.

Fig. 2. (Color online) Projections of crystallization areas in Zr-rich corner of systems: $\mathrm{Zr}-\mathrm{Be}-\mathrm{Fe}$ (a); $\mathrm{Zr}-\mathrm{Cu}-\mathrm{Fe}$ (b). Area of low-melting alloys marked with red colour. 


\section{3. Свойства и возможности применения легкоплавких циркониевых сплавов}

Температура плавления всех групп сплавов приведена в табл. 1, свойства характерного сплава системы $\mathrm{Zr}-\mathrm{Fe}-\mathrm{Be}$ приведены в табл. 3 .

Благодаря наличию большой объемной доли интерметаллидных фаз в структуре сплавов температурный коэффициент линейного расширения и теплопроводность сплавов оказались выше, чем у нелегированного циркония. В то же время коррозионная стойкость в воде и перегретом паре высоких параметров оказалась относительно высокой, свойственной циркониевым сплавам (см. табл. 3).

Ввиду низкой температуры плавления сплавы изготавливались не дуговой плавкой, как обычные циркониевые сплавы, а индукционной плавкой в тиглях из графита или $\mathrm{ZrO}_{2}$. Температура плавки при этом не превышала $1300^{\circ} \mathrm{C}$, так как в образующейся в начале плавки легкоплавкой эвтектике постепенно растворялись более тугоплавкие компоненты. Взаимодействия с материалом тигля при этих температурах не происходило. Внешний вид слитков сплавов при литье в разные изложницы представлен на рис. За. Максимальный вес слитка составлял 15 кг.

Благодаря низкой температуре плавления, хорошим литейным свойствам и слабому взаимодействию с материалом тигля и изложницы сплавы могут применяться как литейные для изготовления изделий сложного профиля (см. рис. 3b).

Табл. 2. Нонвариантные реакции в циркониевом углу систем $\mathrm{Zr}-\mathrm{Fe}, \mathrm{Zr}-\mathrm{Cu}, \mathrm{Zr}-\mathrm{Cu}-\mathrm{Fe}, \mathrm{Zr}-\mathrm{Be}, \mathrm{Zr}-\mathrm{Be}-\mathrm{Fe}$.

Table 2. Nonvariant reactions in zirconium in Zr-rich corner of systems $\mathrm{Zr}-\mathrm{Fe}, \mathrm{Zr}-\mathrm{Cu}, \mathrm{Zr}-\mathrm{Cu}-\mathrm{Fe}, \mathrm{Zr}-\mathrm{Be}, \mathrm{Zr}-\mathrm{Be}-\mathrm{Fe}$.

\begin{tabular}{|c|c|c|c|c|}
\hline 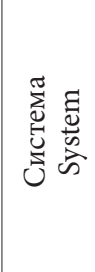 & 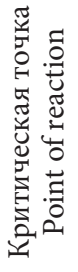 & $\begin{array}{c}\text { Реакция } \\
\text { Nonvariant reaction }\end{array}$ & 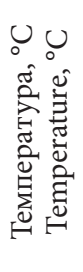 & 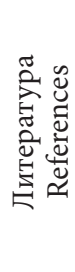 \\
\hline \multirow{2}{*}{ Zr-Fe } & $\mathrm{E}_{1}$ & $\mathrm{~L} \leftrightarrow \beta-\mathrm{Zr}+\mathrm{Zr}_{2} \mathrm{Fe}$ & 928 & {$[6]$} \\
\hline & $\mathrm{P}_{1}$ & $\mathrm{~L}+\mathrm{ZrFe}_{2} \leftrightarrow \mathrm{Zr}_{2} \mathrm{Fe}$ & 974 & {$[6]$} \\
\hline $\mathrm{Zr}-\mathrm{Cu}$ & $\mathrm{E}_{2}$ & $\mathrm{~L} \leftrightarrow \beta-\mathrm{Zr}+\mathrm{Zr}_{2} \mathrm{Cu}$ & 995 & {$[6]$} \\
\hline \multirow{3}{*}{$\begin{array}{l}\mathrm{Zr}-\mathrm{Cu}- \\
\mathrm{Fe}\end{array}$} & $\mathrm{E}_{3}$ & $\mathrm{~L} \leftrightarrow \mathrm{ZrFe}_{2}+\mathrm{Zr}_{2} \mathrm{Cu}$ & 950 & * \\
\hline & $\mathrm{P}_{2}$ & $\mathrm{~L}+\mathrm{ZrFe}_{2} \leftrightarrow \mathrm{Zr}_{2}(\mathrm{Fe}, \mathrm{Cu})+\mathrm{Zr}_{2} \mathrm{Cu}$ & 920 & * \\
\hline & $\mathrm{E}_{4}$ & $\mathrm{~L} \leftrightarrow \beta-\mathrm{Zr}+\mathrm{Zr}_{2}(\mathrm{Fe}, \mathrm{Cu})+\mathrm{Zr}_{2} \mathrm{Cu}$ & 857 & * \\
\hline \multirow{2}{*}{$\mathrm{Zr}-\mathrm{Be}$} & $\mathrm{P}_{4}$ & $\mathrm{~L}+\beta-\mathrm{Zr} \leftrightarrow \alpha-\mathrm{Zr}$ & 975 & [7] \\
\hline & $\mathrm{E}_{5}$ & $\mathrm{~L} \leftrightarrow \alpha-\mathrm{Zr}+\mathrm{ZrBe}_{2}$ & 865 & {$[7]$} \\
\hline \multirow{3}{*}{$\begin{array}{l}\mathrm{Zr}-\mathrm{Be}- \\
\mathrm{Fe}\end{array}$} & $\mathrm{P}_{3}$ & $\mathrm{~L}+\mathrm{ZrFe}_{2} \leftrightarrow \mathrm{Zr}_{2}(\mathrm{Fe}, \mathrm{Be})+\mathrm{ZrBe}_{2}$ & 850 & *, [8] \\
\hline & $\mathrm{P}_{5}$ & $\mathrm{~L}+\beta-\mathrm{Zr} \leftrightarrow \mathrm{Zr}_{2}(\mathrm{Fe}, \mathrm{Be})+\alpha-\mathrm{Zr}$ & 850 & $*$, [8] \\
\hline & $\mathrm{E}_{6}$ & $\mathrm{~L} \leftrightarrow \alpha-\mathrm{Zr}+\mathrm{Zr}_{2}(\mathrm{Fe}, \mathrm{Be})+\mathrm{ZrBe}_{2}$ & 790 & *, [8] \\
\hline
\end{tabular}

Примечание: * - настоящая работа

Comments: ${ }^{*}$ - reference to this article
Сплавы также изготавливались в виде гранул (для технологии капиллярной пропитки) или аморфных лент (см. рис. 3c, 3d). При быстрой закалке сплавы, особенно сплавы системы $\mathrm{Zr}-\mathrm{Ti}-\mathrm{Fe}-\mathrm{Be}-\mathrm{Cu}$, легко аморфизируются. Максимальная полученная толщина аморфной ленты составляла 250 мкм, а ширина 50 мм.

Сплавы обладают высокими капиллярными свойствами. Две циркониевые плотно сжатые пластины с прорезями толщиной от 0.05 до 0.35 мм были опущены в расплав сплава и выдержаны при температуре более $910^{\circ} \mathrm{C}$ в течение нескольких минут. За счет капиллярных сил расплав поднялся на высоту до 50 мм, причем затек даже в места, где зазора практически не было (см. рис. 4).

Сплавы в виде аморфных лент использовались также для пайки разнородных материалов - керамики с металлом, графита с металлом, пропитки пористого графита и т. д. (см. рис. 5). Во всех случаях припой самопроизвольно затекал в микронные зазоры.

\section{4. Возможности применения легкоплавких циркониевых сплавов в атомной энергетике}

Дисперсионый тепловыделяющий элемент (твэл) представляет собой заключенный в оболочку сердечник, в котором частицы ядерного топлива распределены в неделящемся матричном материале [9]. Дисперсионные твэлы обладают высокой радиационной стойкостью, надежностью, работоспособностью в режиме переменных нагрузок, способны достигать высоких выгораний.

табл. 3. Свойства сплава, содержащего 6.0\% масс. железа, 2.7\% бериллия, цирконий остальное.

Table 3. Properties of alloy containing $6.0 \%$ mass. Fe and $2.7 \% \mathrm{Be}$, remainder $-\mathrm{Zr}$.

\begin{tabular}{|c|c|c|}
\hline $\begin{array}{l}\text { Характеристика } \\
\text { Feature }\end{array}$ & $\begin{array}{l}\text { Температура, }{ }^{\circ} \mathrm{C} \\
\text { Temperature, }{ }^{\circ} \mathrm{C}\end{array}$ & $\begin{array}{c}\text { Значение } \\
\text { Value }\end{array}$ \\
\hline $\begin{array}{l}\text { Плотность, г/ } \mathrm{cm}^{3} \\
\text { Density, g/ } / \mathrm{cm}^{3}\end{array}$ & 20 & $6,1-6,3$ \\
\hline $\begin{array}{c}\text { Температурный коэффициент } \\
\text { линейного расширения, } \mathrm{K}^{-1} \\
\text { Temperature coefficient } \\
\text { of linear expansion, } \mathrm{K}^{-1}\end{array}$ & $100-500$ & $(9-11) \cdot 10^{-6}$ \\
\hline $\begin{array}{l}\text { Скорость коррозии } \\
\text { в воде и паре, г/ } \text { м }^{2} ч\end{array}$ & 350 & $0,002-0,004$ \\
\hline $\begin{array}{l}\text { Velocity of corrosion } \\
\text { in the water and steam, } g / \mathrm{m}^{2} \mathrm{~h}\end{array}$ & 550 & $0,2-0,5$ \\
\hline \multirow{3}{*}{$\begin{array}{l}\text { Теплопроводность, Вт/м·K } \\
\text { Thermal conductivity, } \mathrm{W} / \mathrm{m} \cdot \mathrm{K}\end{array}$} & 100 & $12-13$ \\
\hline & 300 & $16-18$ \\
\hline & 500 & $22-26$ \\
\hline \multirow{3}{*}{$\begin{array}{c}\text { Прочность при сжатии, МПа } \\
\text { Compressive strength, } \mathrm{MPa}\end{array}$} & 100 & $700 \pm 50$ \\
\hline & 300 & $650 \pm 50$ \\
\hline & 500 & $500 \pm 50$ \\
\hline
\end{tabular}




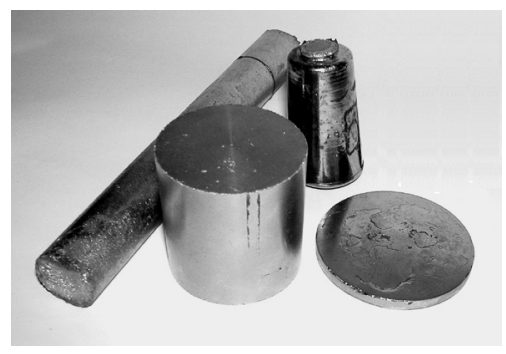

a

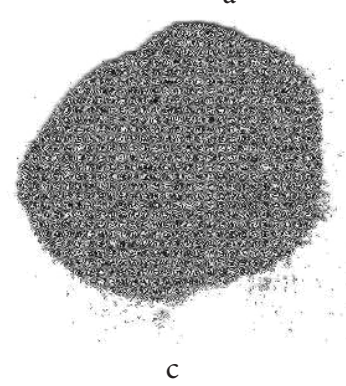

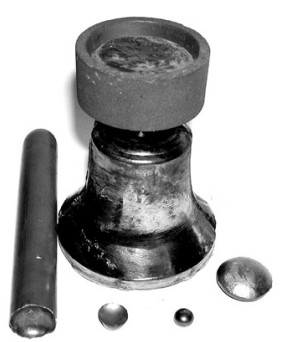

b

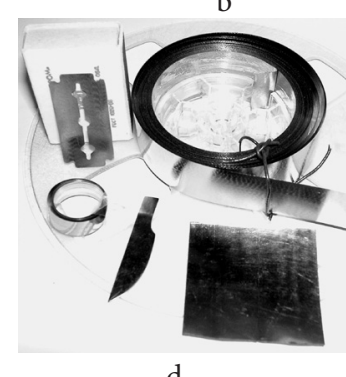

Рис. 3. Внешний вид слитков сплавов системы $\mathrm{Zr}-\mathrm{Fe}-\mathrm{Cu}$ (a), литых изделий из сплава $\mathrm{Zr}-\mathrm{Fe}-\mathrm{Be}-\mathrm{Cu}(\mathrm{b})$, гранул из сплава системы $\mathrm{Zr}-\mathrm{Ti}-\mathrm{Fe}-\mathrm{Cu}-\mathrm{Be}$ (c), аморфных лент и аморфных изделий из сплава системы $\mathrm{Zr}-\mathrm{Ti}-\mathrm{Fe}-\mathrm{Cu}-\mathrm{Be}(\mathrm{d})$.

Fig. 3. Appearance of ingots of alloys of $\mathrm{Zr}-\mathrm{Fe}-\mathrm{Cu}$ (a); the molded products of alloys of $\mathrm{Zr}-\mathrm{Fe}-\mathrm{Be}-\mathrm{Cu}$ (b) granules of alloys of $\mathrm{Zr}-\mathrm{Ti}-\mathrm{Fe}-\mathrm{Cu}-\mathrm{Be}$ (c), the amorphous ribbons and amorphous products of alloys of $\mathrm{Zr}-\mathrm{Ti}-\mathrm{Fe}-\mathrm{Cu}-\mathrm{Be}(\mathrm{d})$.

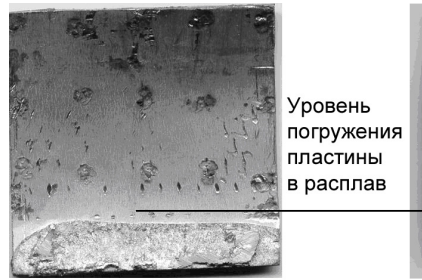

a

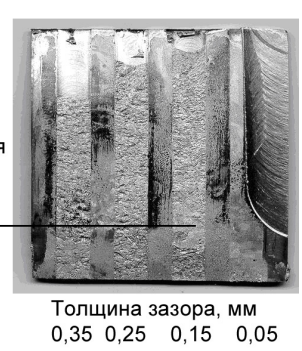

b
Pис. 4. Капиллярные свойства сплава $\mathrm{Zr}-\mathrm{Fe}-\mathrm{Cu}$ при $910^{\circ} \mathrm{C}$ : внешняя (a) и внутренняя (b) плоскости пластины.

Fig. 4. Capillary properties of $\mathrm{Zr}-\mathrm{Fe}-\mathrm{Cu}$ alloy at $910^{\circ} \mathrm{C}$ : outer (a) and inner (b) surfaces of the plate.

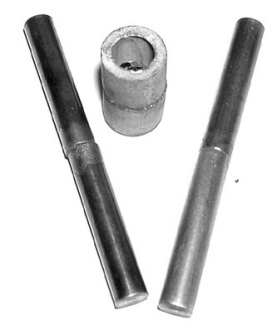

Рис. 5. Пайка материалов припоем из аморфной ленты сплава системы $\mathrm{Zr}-\mathrm{Ti}-\mathrm{Fe}-\mathrm{Be}-\mathrm{Cu}$; соединения, слева направо: $\mathrm{Zr}-\mathrm{Zr}$, $\mathrm{Al}_{2} \mathrm{O}_{3}-\mathrm{Al}_{2} \mathrm{O}_{3}$, $\mathrm{Zr}$ - нержавеющая сталь.

Fig. 5. Soldering with solder from amorphous band of $\mathrm{Zr}-\mathrm{Ti}-\mathrm{Fe}-$ $\mathrm{Be}-\mathrm{Cu}$ alloy; connections, from left to right: $\mathrm{Zr}-\mathrm{Zr}, \mathrm{Al}_{2} \mathrm{O}_{3}-\mathrm{Al}_{2} \mathrm{O}_{3}$, Zr-stainless steel.
Главные требования к матричному материалу: хорошая совместимость с топливными частицами и оболочкой, высокая коррозионная и радиационная стойкость, возможно более низкое значение сечения захвата тепловых нейтронов, относительно низкая температура плавления. Разработанные легкоплавкие циркониевые сплавы обладают всеми указанными свойствами (см. табл. 1, 3, 4) [10].

Для изготовления дисперсионных твэлов с использованием легкоплавких циркониевых сплавов в качестве матричных был разработан метод капиллярной пропитки, заключающийся в том, что циркониевый матричный сплав в виде гранул в смеси с гранулами топлива засыпается в оболочку твэла, а затем нагревается до температуры на $50^{\circ} \mathrm{C}$ превышающей температуру плавления сплава. При этом расплав циркониевого сплава под действием капиллярных сил покрывает гранулы топлива и оболочку и затекает в стыки между топливными гранулами и оболочкой, образуя так называемые «мостики», что обеспечивает сохранение высокой теплопроводности топливного сердечника (см. рис. 6). Благодаря высоким капиллярным свойствам матричный сплав в про-

Табл. 4. Слой взаимодействия после отжига сплава системы $\mathrm{Zr}-\mathrm{Fe}-\mathrm{Be}(-\mathrm{Cu})$ с различными топливными композициями в течение 6000 ч при температуре $750^{\circ} \mathrm{C}$.

Table 4. Interaction layer after annealing $\mathrm{Zr}-\mathrm{Fe}-\mathrm{Be}(-\mathrm{Cu})$ alloy with various fuel composition at $750^{\circ} \mathrm{C}$ for $6000 \mathrm{~h}$.

\begin{tabular}{|c|c|}
\hline $\begin{array}{c}\text { Топливная композиция } \\
\text { Fuel composition }\end{array}$ & $\begin{array}{c}\text { Толщина слоя, мкм } \\
\text { Layer width, } \mu \mathrm{m}\end{array}$ \\
\hline $\mathrm{UO} 2+(\mathrm{Zr}-\mathrm{Fe}-\mathrm{Be})$ & 25 \\
\hline$(\mathrm{U} 5 \mathrm{Nb} 5 \mathrm{Zr})+(\mathrm{Zr}-\mathrm{Fe}-\mathrm{Be}-\mathrm{Cu})$ & 10 \\
\hline $\mathrm{U}_{3} \mathrm{Si}+(\mathrm{Zr}-\mathrm{Fe}-\mathrm{Be}-\mathrm{Cu})$ & 10 \\
\hline $\mathrm{U}_{9} \mathrm{Mo}+(\mathrm{Zr}-\mathrm{Fe}-\mathrm{Be}-\mathrm{Cu})$ & 10 \\
\hline
\end{tabular}

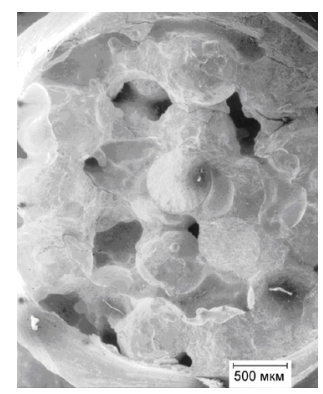

a

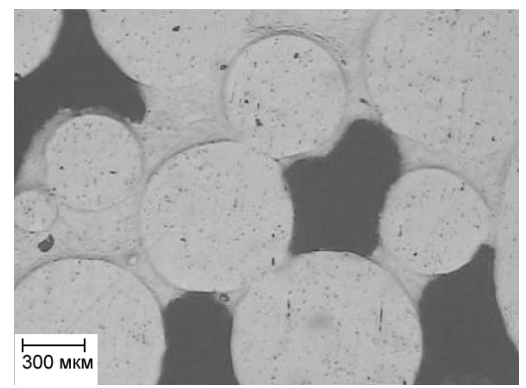

b
Рис. 6. Макроструктура (а) и микроструктура (b) топливного сердечника, изготовленного методом капиллярной пропитки).

Fig. 6. Macrostructure (a) and microstructure (b) of fuel composition made by capillary impregnation method. 
цессе расплавления при изготовления твэла не только покрывает гранулы топлива, но и также внутреннюю поверхность оболочки, полностью защищая ее от взаимодействия с топливом.

Используя в качестве топливных частиц сплавы U9Mo, U-3Nb-1.5Zr, U-1.5Mo-1.0Zr, а в качестве матричных - легкоплавкие циркониевые сплавы, можно получить содержание урана под оболочкой твэла $10,7-12,9$ г/ $\mathrm{cm}^{3}$, что на $30-50 \%$ выше, чем у штатного твэла ВВЭР-1000 и PWR.

Основные преимущества применения дисперсионного топлива с циркониевыми матричными сплавами:

- высокая теплопроводность топливного сердечника 18-20 Вт/(м·К), что снижает максимальную температуру топлива до $450-500^{\circ} \mathrm{C}$, реализует концепцию «холодного» ядерного топлива и повышает эксплуатационную надежность и безопасность АЭС;

- предложенная технология дает возможность обеспечить регулируемую пористость в топливном сердечнике, что позволяет компенсировать распухание топлива до выгорания 120 МВт·сут/т U, увеличить кампанию твэлов, уменьшить объемы отработавшего топлива, экономить природный уран;

- наличие металлургического сцепления между оболочкой и сердечником делает твэлы работоспособными в режиме переменных нагрузок, что приведет к повышению их эксплуатационной надежности и безопасности АЭС;

- высокая коррозионная стойкость в воде высоких параметров;

- защита оболочки от взаимодействия с металлическим топливом и осколками деления при возможном применении топливных композиций для быстрых реакторов;

- высокая ураноемкость.

\section{5. Выводы}

Проведен анализ возможностей и особенностей использования циркониевых сплавов, представляющих собой глубокие тройные и четверные эвтектики с очень низкими для циркония температурами плавления от 690 до $860^{\circ} \mathrm{C}$, а также модификаций этих сплавов. Сплавы обладают высокими капиллярными свойствами, могут использоваться как литейные, могут применяться для пайки разнородных материалов, могут использоваться в аморфном состоянии в виде гранул и лент.

Перспективно использование легкоплавких циркониевых сплавов в атомной энергетике в качестве матричных сплавов дисперсионных твэлов. Топливные композиции, включающие эти сплавы, обладают высокой теплопроводностью, совместимостью и ураноемкостью на 30 - 50\% выше, чем для твэлов реакторов ВВЭР и PWR.

\section{Литература/References}

1. A. S. Zaimovsky, A.V. Nikulina, N.G. Reshetnikov. Zirconium alloys in atomic energy. Moscow, Energoatomizdat (1994) 252 p. (in Russian) [А. С. Займовский, А. В. Никулина, Н. Г. Решетников. Циркониевые сплавы в ядерной энергетике. М.: Энергоатомиздат, 1994. 252 с.]

2. D.L. Douglass. Metallurgy of zirconium. Vienna. International Atomic Energy Agency (1971) (in Russian) [Д. Дуглас. Металловедение циркония. Пер. с англ. под ред. А. С. Займовского М.: Металлургиздат, 1975, 360 c.]

3. A. V. Nikulina. Zirconium alloys in atomic energy. Metal science and heat treatment, 11, 8-12, 2004 (in Russian) [А.В. Никулина Циркониевые сплавы в атомной энергетике. Металловедение и термическая обработка металлов, 11, 2004, с. 8 - 12]

4. A. Savchenko, I. Konovalov, A. Vatulin, A. Morozov, V. Orlov, O. Uferov, S. Ershov, A. Laushkin, G. Kulakov, S. Maranchak, Z. Petrova. Dispersion type zirconium matrix fuels fabricated by capillary impregnation method. J. of Nucl. Mater., 362, 356-363 (2007).

5. A.M. Savchenko, A.V. Vatulin, A.V. Morozov, G.V. Kulakov, S.A. Ershov, A.V. Laushkin, S. V. Maranchak, Y.V. Konovalov, E. K. Malamanova. Zirconium matrix alloys as innovative material for different types of fuel. Progr. in Nucl. Energy, 2012, 57, $138-144$ (2012)

6. Binary Alloy Phase Diagrams. Handbook. Ed. by N.P. Lyakishev. V. 2. Moscow, Mashinostroenie (1997) 1024 p. (in Russian) [Диаграммы состояния двойных металлических систем: Справочник. В 3-х т.: Т. 2 под общей редакцией Н.П. Лякишева. - М.: Машиностроение, 1997, 1024 с.]

7. Binary Alloy Phase Diagrams. Handbook. Ed. by N.P. Lyakishev. V. 1. Moscow, Mashinostroenie (1996) 992 p. (in Russian) [Диаграммы состояния двойных металлических систем: Справочник. В 3-х т.: Т. 1 под общей редакцией Н.П. Лякишева. - М.: Машиностроение, 1996, 992 с.]

8. Physical chemistry of zirconium alloys. Ed. by O.S. Ivanov. Moscow, Nauka (1968) 277 p. (in Russian) [Физико-химия сплавов циркония. Под редакцией О. С. Иванова. М.: Наука, 1968, 277 с.]

9. A.G. Samoilov, A.I. Kashtanov, V.S. Volkov. Dispersion fuel elements. Materials and technology. Moscow, Energoatomizdat (1982) 224 p. (in Russian) [А. Г..Самойлов, А.И. Каштанов, В.С. Волков Дисперсионные твэлы. Материалы и технология. М.: Энергоатомиздат, 1982. 224 с.]

10. A. Savchenko, A. Vatulin, I. Konovalov, A. Morozov, V. Sorokin, S. Maranchak. Fuel of Novel Generation for PWR and as Alternative to MOX Fuel. J. of Energy Conversion \& Management, 51, 1826 - 1833 (2010) 